\title{
Neuromarketing y Memoria: Implicaciones para la Comunicación Publicitaria
}

\author{
Nuria SERRANO ABAD ${ }^{1}$ \\ Cristina DE BALANZÓ BONO ${ }^{2}$ \\ Blanquerna - Universitat Ramon Llull
}

\begin{abstract}
RESUMEN:
El estudio de la memoria es relevante y fundamental en relación con la actividad y la investigación publicitaria. Sin embargo, a pesar de lo mucho que hemos aprendido sobre la memoria a lo largo de los años, aún no entendemos completamente cuál es su papel en conseguir una publicidad más eficaz. Desde nuestro punto de vista, este contexto cambiará gracias a reveladoras aportaciones de la neurociencia cognitiva y el desarrollo del neuromarketing. Este artículo recoge un estado de la cuestión que describe estas iniciales contribuciones, como punto de partida para otros investigadores interesados en este apasionante campo.
\end{abstract}

PALABRAS CLAVE: Memoria; Neuromarketing; Publicidad; Neurociencia Cognitiva; Investigación de mercados.

TITLE: Neuromarketing and memory: Implications for Advertising

\begin{abstract}
:
Memory is a relevant and fundamental part of advertising and advertising research. It is usually obvious to include the study of memory on any given research project. But despite everything we have learned about memory throughout the years, we still haven't completely understood how it influences advertising effectiveness. Our point of view is that this is about to change thanks to relevant findings from cognitive neuroscience, and the development of neuromarketing. This article summarizes current findings on the subject as a starting point for other researchers in the field.
\end{abstract}

KEY WORDS: Memory; Neuromarketing; Advertising; Cognitive Neuroscience; Market Research.

\section{Introducción}

Se calcula que nuestra memoria retiene más de 10.000 marcas (Franzen y Bouwman, 2001) que conocemos, que amamos, que odiamos, que acumulamos como experiencias, y que asociamos a algunos fragmentos de sus acciones de co-

1 Licenciada en Psicología por la Universidad Central de Barcelona y doctorando en la Facultad de Ciencias de la Comunicación Blanquerna - Universidad Ramón Llull de Barcelona, donde es miembro del Grupo de Investigación en Estrategia y Creatividad Publicitarias. Es Directora de Planificación Estratégica en la agencia VCCP Spain. E-mail: nurserrano@yahoo.com. Twitter: @neuroadvertisng

2 Doctora en Publicidad y Licenciada en Sociología por la Universidad Autónoma de Barcelona. Es miembro del Grupo de Investigación en Estrategia y Creatividad Publicitarias de la Universidad Ramon Llull de Barcelona. Es Global Head of Neuroscience en TNS en el Reino Unido. Email: Cristina.debalanzo@tnsglobal.com. Twitter:@crisbalanzo 
municación, de sus anuncios, de sus melodías, de experiencias que hemos vivido. Quizás por ello, el gran reto para los investigadores publicitarios y para el marketing sea cómo crear e influir en el recuerdo y obtener así un lugar privilegiado en la mente del consumidor.

En este trabajo, hemos realizado un análisis teórico sobre la memoria y su papel en el ámbito de la creación, la estrategia y la investigación publicitaria, desde el enfoque de las neurociencia cognitiva y el neuromarketing. Existen diversos artículos académicos sobre el neuromarketing a modo general, incluso en español (Balanzó y Sabaté, 2007 y Monge y Fernández, 2011), pero no se ha realizado un artículo académico en español que ofrezca un completo estado de la cuestión sobre las aportaciones de la neurociencia cognitiva y el neuromarketing en relación con la memoria. Por esa razón, hemos creído necesario llevar a cabo un análisis que describa las principales aportaciones científicas que ofrecen una nueva visión sobre la memoria y su influencia en el proceso publicitario, haciéndonos reflexionar sobre la relevancia de las actuales reglas de la estrategia y la creación publicitaria, así como reconsiderar las bases actuales de medición de la eficacia en la comunicación publicitaria.

La neurociencia cognitiva fusiona los avances producidos por las ciencias que estudian el sistema nervioso, como la neurobiología o la neurofarmacología, con los conocimientos obtenidos por la psicología cognitiva. Es una disciplina que se desarrolla a finales del siglo XX, pero que adquiere gran relevancia en el siglo XXI. Además de la memoria, la neurociencia cognitiva investiga la influencia de otros procesos cognitivos como la percepción, la atención, el razonamiento, la toma de decisiones o la representación del objeto y la emoción. El desarrollo actual de la neurociencia cognitiva viene de la mano de los nuevos avances tecnológicos que han permitido visualizar y medir el comportamiento biológico neuronal de nuestra actividad cerebral durante esos procesos.

Existen, y siguen apareciendo, teorías acerca de los mecanismos que subyacen en la memoria, pero es la neurociencia cognitiva una de las disciplinas que está aportando más luz sobre su funcionamiento. El neuromarketing consiste en el desarrollo y la aplicación de las técnicas de la neurociencia cognitiva a la investigación de marketing tradicional (Monte, 2011). Representa el encuentro entre el conocimiento médico, las tecnologías y el marketing para estudiar las reacciones del cerebro ante ciertos estímulos como la marca o el producto y profundizar en el conocimiento de las necesidades, motivaciones, deseos o aspiraciones (Blanco, 2011).

\section{Principales características de la memoria desde la perspectiva del neuro- marketing}

La base científica de la neurociencia cognitiva y, por tanto, el punto de partida del neuromarketing, hace referencia a que el funcionamiento del cerebro es más cercano a un ecosistema biológico activo que a la habitual metáfora del ordenador 
(Ratey, 2004). Cuando se observa el cerebro a través de instrumentos biométricos como los escáneres PET, se comprueba que su funcionamiento está muy alejado de una programación estática y pre-organizada. En ese sentido, no existe una única área para la visión, el lenguaje, la emoción, ni siquiera para la memoria. Es totalmente incorrecta la idea de que las memorias se guardan y se almacenan como si de archivos se tratase. De hecho, el funcionamiento cerebral en general y, específicamente, el de la memoria, consiste en la interrelación de un complejo y sofisticado grupo de sistemas cerebrales.

A continuación, veremos cómo, desde la neurociencia cognitiva, se redefine la memoria, aportando una nueva manera de entenderla, valorarla y medirla, desde donde se abre un mundo de oportunidades en investigación.

\subsection{Redefinición del concepto de memoria}

Hasta la irrupción de los nuevos hallazgos de la neurociencia cognitiva, lo habitual había sido considerar la memoria como si se tratase de «información» (más o menos concreta y más o menos «archivada») que, en la mayoría de casos, se podía rescatar mediante preguntas directas. Ese punto de partida llevaba a considerar que las respuestas eran objetivas y su medición, en principio, relativamente simple. Una base sobre la que se han asentado históricamente los conceptos sobre la memoria, incluso en publicidad.

Sin embargo, esta definición no está en línea con los nuevos descubrimientos de la neurociencia cognitiva, ya que limita su verdadero significado y sus funciones. Es por ello que se requiere una nueva definición de la memoria, más adecuada a los nuevos tiempos, que aglutine los nuevos descubrimientos científicos y concrete sus principales características.

En ese sentido, la primera de las características que definen a la memoria se fundamenta en la evidencia científica de que las imágenes no se archivan como facsímiles de cosas, eventos, palabras o frases. No existen imágenes permanentes de nada, ni siquiera en versión en miniatura, lo que contradice totalmente la creencia que la memoria sea un almacén de recuerdos (Damasio, 2005). Por lo tanto, no podemos seguir pensando que las memorias son como fotos de un álbum que si las «guardamos» adecuadamente se recordarán bajo las mismas condiciones (Schacter, 2001). Lo que sucede en realidad, aunque todavía sea difícil de describir detalladamente, es que las memorias son estados de la actividad espacio-temporal del sistema nervioso y, por lo tanto, sería difícil que se pudieran archivar en el tiempo como tales (Dudai, 2004).

Por consiguiente, la nueva definición de memoria pasa por comprender que no almacenamos en sentido literal y este cambio de concepción creemos que podría generar importantes implicaciones para la publicidad.

La segunda de las características de la memoria es que siempre está activa, siempre está preparada para recibir el flujo interminable de percepciones (Ratey, 
2004). Gracias a los estudios realizados sobre el hipocampo, se ha podido demostrar que no podemos activarlo o desactivarlo a voluntad. El hipocampo actúa igualmente tanto cuando estamos intentando memorizar algo, como cuando estamos simplemente leyendo o viendo algo sin la intención de memorizarlo. Esto se ha podido comprobar midiendo la actividad del hipocampo, diferenciando entre personas que intentan memorizar listas de palabras y comparándolo con personas que únicamente leían las mismas listas (Eichenbaum, 2003).

Otra característica de la memoria es que es flexible y adaptativa. Esto es posible porque los sistemas biológicos que componen nuestro cerebro no son rígidos ni se basan en compartimentos estancos. Anteriormente, la mayoría de científicos consideraban que el sistema nervioso adulto no se modificaba, exceptuando el caso de las degeneraciones neuronales. Fue en 1958 cuando se demostró que el sistema nervioso mantiene toda la vida la capacidad de modificación anatómica y funcional (Sampedro, 1966). En estudios más recientes, también se ha podido evidenciar que los cambios cerebrales existen, incluso en relación con aspectos tan vinculados a la comunicación como es la utilización de redes sociales en Internet. En un experimento publicado por la revista Nature Neuroscience, se demuestra la relación del tamaño de la amígdala cerebral de los individuos en función del tamaño y complejidad de su red social en Internet (Bickart, 2011).

De hecho, nuestro cerebro plástico se está modificando incluso mientras estamos leyendo este texto; podríamos decir que, cada mañana, nos levantamos con un cerebro nuevo. Para ello, el rol de la memoria es fundamental, ya que si la memoria fuera absolutamente estática y almacenáramos la información de un modo lineal, sería imposible que el cerebro cambiara naturalmente, o lo que es peor, sería imposible que aprendiéramos nada. La flexibilidad de la memoria es una característica fundamental para el desarrollo del ser humano: sus aptitudes y su adaptación a los diferentes entornos dependen en gran medida de esta flexibilidad.

La cuarta característica de la memoria es que no se ubica en una zona determinada del cerebro (Schacter, 1996), aunque sí hay zonas cerebrales que tienen una mayor relación con este proceso cognitivo. Cada vez que aprendemos algo nuevo, como decíamos, nuestro cerebro se modifica y esto es así en el sentido neuronal, pero también desde el punto de vista espacial. El entorno total del cerebro se modifica de modo constante, espontáneamente o voluntariamente (Damasio, 2010). En relación con este punto, es importante destacar que, biológicamente, el ser humano está diseñado tanto para recordar como para olvidar. Tener una memoria extraordinaria no es beneficioso, como se ha podido comprobar con sujetos que tenían dicha disfunción, ya que les imposibilitaba llevar una vida normal.

Como conclusión a este primer epígrafe, podemos afirmar que, desde la neurociencia cognitiva y el neuromarketing, emerge una nueva manera de entender el funcionamiento de la memoria. Una memoria que se define a partir de las siguientes características básicas: no literal, activa, flexible, adaptativa y dinámica. 
Todo ello nos invita a hacer una primera reflexión ya que, en publicidad, más allá de si se recuerdan o no la marca y los mensajes, quizás deberíamos obtener más información sobre cómo se modifican nuestro cerebro y nuestras memorias, y en qué modo puede esto influir en el consumidor y en la eficacia publicitaria en un corto y largo plazo.

\subsection{La memoria es subjetiva}

Además de las características básicas de la memoria anteriormente definidas, hay una serie de particularidades que exponen una nueva variable fundamental: la memoria, según los descubrimientos de la neurociencia cognitiva, es subjetiva.

En primer lugar, esa subjetividad se manifiesta en que los recuerdos se modifican en el momento en que los recordamos, y, por tanto, también cada vez que los volvemos a recordar. La teoría constructivista ya defendía que las memorias se construían de información del pasado junto con información generada en el momento del recuerdo y, posteriormente, esto se ha podido probar desde las neurociencias. El contenido de la memoria se podría considerar, entonces, interactivo, ya que la información incorporada por el estímulo inmediato interactúa con información anterior que ya tiene el sujeto, siendo el conjunto lo que configura el contenido final recordado (Howes, 2007). Incluso, en el caso de que hayamos olvidado alguno de los elementos del recuerdo, en el momento de intentar acordarnos podemos completar la información desde el nuevo estímulo. Como resultado, las memorias siempre serán diferentes, subjetivas y cambiantes.

Por consiguiente, la memoria y la construcción del recuerdo se han de entender como sistemas dinámicos e interactivos y esto para la publicidad reviste una gran importancia. Por ejemplo, es una característica a tener en cuenta para la planificación de medios, ya que es de esperar que un proceso similar suceda después de cada visionado repetido de un anuncio. Si asumimos que cada vez que recordamos un evento se activan diferentes áreas del cerebro, seguro que también ocurrirá cada vez que veamos el mismo anuncio. El receptor está activo y aporta información de visionados y experiencias previas. Por lo tanto, éstas también deben tenerse en cuenta cuando analizamos y medimos el recuerdo sobre un estímulo específico. Por ejemplo, en una investigación de post-test, deberemos tener en cuenta que el receptor estará recordando un conjunto de información que incluye los visionados anteriores, lo que está sucediendo en ese momento, y que, incluso, la manera en que hemos formulado la pregunta sesgará tal recuerdo.

En segundo lugar, destacamos la dimensión subjetiva y personal de la memoria, ya que cada persona recuerda los hechos bajo su desarrollo cerebral particular. Si nos paramos a pensar por un momento en nuestras propias experiencias, nos sobrarán ejemplos en los que, al explicar una anécdota vivida en grupo, cada una de las personas que estuvieron presentes la desarrollaría de una manera diferente. En función de nuestra personalidad, es posible que hayamos pensado que los demás son 
más fantasiosos que nosotros, que quizás no prestaron suficiente atención, o que uno mismo puede estar haciéndose viejo. Este tipo de razonamiento es contrario a lo que la neurociencia cognitiva defiende. La existencia de la subjetividad en la memoria es verídica y viene determinada, en primer lugar, por el proceso de percepción, que en sí mismo ya es subjetivo: todos vemos, oímos, olemos, tocamos y saboreamos de una manera personal. Y, por otro lado, viene influenciado también por los propios recuerdos del pasado, como hemos descrito anteriormente. Por lo tanto, la memoria de cualquier evento refleja cómo éste ha sido percibido y entendido por el individuo de un modo personal y único (Howes, 2007).

Nuestras memorias están siendo influenciadas constantemente por nuestra historia, nuestras creencias y nuestro conocimiento. Una memoria perfecta y fiable es un mito (Damasio, 2010). En el caso de la publicidad, este factor nos obliga a reflexionar sobre el rol que tiene en el recuerdo actual cualquier experiencia pasada que hayamos tenido con el producto, la marca, o con campañas similares de algún modo más o menos identificables, como también lo es el contexto en el que tiene lugar la comunicación, así como el momento personal en que estamos recibiendo un estímulo determinado.

La última característica de la memoria que vamos a destacar es que ésta es subjetiva y diferente a la de los demás, y, por tanto, es una memoria que se equivoca. La neurociencia cognitiva defiende que todos tenemos memorias falsas, es un resultado biológico que nos define como humanos (Ratey, 2004). Aunque pueda sonar desconcertante, sucede así porque evolutivamente estamos diseñados para que así sea. Equivocarnos nos permite adaptarnos a un medio ambiente que cambia constantemente (Damasio, 2010).

Pongamos un ejemplo de la vida cotidiana y se entenderá a lo que nos estamos refiriendo de un modo sencillo. Cada vez que aparcamos el coche en el aparcamiento del supermercado al que vamos habitualmente, realizamos un complejo esfuerzo de memoria. Por una parte, memorizamos dónde lo hemos aparcado, por otra, deberemos recordar dónde lo hemos aparcado cuando salimos de hacer la compra. Pero la memoria también tiene un tercer rol fundamental: olvidarse de todos los otros sitios donde hemos aparcado en el pasado para que no interfieran con el recuerdo actual. Imaginemos lo que supondría acordarnos de todas y cada una de las veces que hemos aparcado el coche en ese aparcamiento.

Por lo tanto podemos afirmar, desde la neurociencia cognitiva y del neuromarketing, que la memoria, además de no literal, activa, flexible, adaptativa y dinámica, es subjetiva, reinterpreta, olvida y se equivoca.

\subsection{Tipos de memoria: memoria a corto y largo plazo}

En un principio, se creyó que la memoria era unitaria, no modular, y fue así hasta que se descubrió que había diferencias estructurales y funcionales cuando se trataba de los recuerdos pasados y nuevos. La observación y experimentación con pa- 
cientes con lesiones en diferentes partes del cerebro ayudó a confirmar que existían al menos dos tipos diferentes de memoria. Uno de los primeros pacientes fue HM, un hombre que sufrió daños en los dos lóbulos temporales (Squire, 1992). Perdió la capacidad de formar nuevos recuerdos, pero conservó todos los recuerdos que tenía antes de su lesión. Esto se llama amnesia anterógrada, la imposibilidad de transferir la información recién adquirida en el corto plazo al largo plazo (a diferencia de la amnesia retrógrada, que es la pérdida de la memoria antes de la lesión o antes de que el trauma tenga lugar). El análisis confirmó que el lóbulo temporal medial, en el hipocampo, era directamente responsable de su incapacidad para formar nuevos recuerdos y de ahí la relación de esta estancia cerebral con la memoria. Estos resultados fueron confirmados por numerosos estudios de casos similares, así como por experimentos realizados con animales (monos y ratas, en su mayoría) con lesiones en el hipocampo (Squire, 1992).

La memoria a corto plazo es ventajosa para el rápido procesamiento cognitivo de los estímulos percibidos, pero, a diferencia de la memoria a largo plazo, tiene una actividad espacio-temporal más limitada. Existen diferentes estimaciones sobre el número de «unidades» o «trozos» de información que se activan en un tiempo determinado, pero éste varía de cuatro (Cowan, 2000) a siete (Miller, 1956). Por supuesto, estas cifras son sólo una estimación, ya que es difícil decidir de manera concluyente lo que es una unidad de información.

La memoria a largo plazo ocupa un área más grande del cerebro en comparación con la memoria a corto plazo y participa activamente en la vida diaria (es decir, vocabulario, navegación o la misma memoria de trabajo). A pesar de su capacidad infinita (en teoría), ésta no es totalmente fiable y se ha demostrado que incluso ante recuerdos muy claros, éstos pueden ser manipulados y reordenados por los recuerdos y/o las percepciones de otros (Braun-Latour y Zaltman, 2006). Por lo general, esto ocurre en la fase de recuperación, como ya describimos en el epígrafe anterior. Este hecho puede convertirse en una ventaja para los anunciantes (aunque también podría ser una desventaja), ya que las opiniones y las actitudes no son inamovibles, se puede lograr cambiar tales recuerdos, un determinado posicionamiento o unas asociaciones con la marca. Como afirmábamos en puntos anteriores, la plasticidad del cerebro y el dinamismo de la memoria explicarían tales fenómenos.

\subsection{Memoria explícita e implícita}

Para un sistema tan complejo como la memoria, no sólo existe una manera de clasificarla. De modo paralelo a la existencia de memorias a corto plazo y a largo plazo, encontramos la memoria explícita y la memoria implícita.

La memoria explícita se describe como la información que una persona puede declarar, como son los hechos y el conocimiento de los acontecimientos vitales específicos que le acaecen a lo largo de su vida. Es el tipo de memoria que se daña 
más a menudo cuando las personas sufren ambos ejemplos de amnesia que hemos citado (Squire, 1992).

La memoria explícita codifica el conocimiento de hechos -nombres, caras, eventos, cosas, y son a las que se accede directamente desde la atención consciente (Ratey, 2004).

Por otra parte, la memoria implícita incluye todas aquellas memorias que no pueden rescatarse conscientemente. Son memorias también llamadas no declarativas, y suponen un procesamiento de información que no requiere atención consciente (Howes, 2007). Éstas incluyen las memorias que surgen de modo involuntario, así como las que agrupan «conocimientos generales» (por ejemplo: tocar un instrumento, el lenguaje, la sintaxis o ir en bicicleta). No somos conscientes de que tenemos este conocimiento, y lo utilizamos de forma automática (Schacter, 1987). La memoria implícita es la responsable de las habilidades y los hábitos que, una vez aprendidos, se realizan sin intención consciente, como comer, hablar, caminar, ir en bicicleta o, incluso, cómo hacer amigos. Son recuerdos inflexibles, lentos de aprender, pero excepcionalmente fiables (Ratey, 2004).

La memoria implícita es menos probable que sea afectada por lesiones o traumas, en parte porque es muy probable que involucre muchas más partes del cerebro de las que se cree. Se ha dado el caso que, incluso, personas que han perdido la memoria explícita aún muestran el reconocimiento de palabras u objetos si se aplican, por ejemplo, técnicas de priming que favorecen el recuerdo que trata de rememorarse. El priming es una técnica (o recurso) que ayuda a recordar y recuperar información a partir del reconocimiento de un material determinado, por ejemplo, proporcionando pistas en forma de una versión de la imagen que a los participantes se les ha pedido que recuerden, o dándoles una palabra que es semánticamente similar.

Como cabe suponer, la memoria implícita, a pesar de ser potencialmente unas de las de mayor interés para el neuromarketing, es muy difícil de demostrar en investigación, incluso cuando los estudios se llevan a cabo bajo estrictas condiciones de laboratorio y en experimentos controlados.

\section{Implicaciones para la comunicación publicitaria}

Una vez definida la memoria desde la nueva perspectiva de la neurociencia cognitiva y el neuromarketing, es importante explorar su complejo funcionamiento en relación con su rol en el proceso publicitario, y encontrar nuevos planteamientos orientados a conseguir una publicidad más efectiva. Es importante destacar que, todavía, no se dispone de metodologías ampliamente aceptadas por la comunidad científica y el número de experimentos publicados aún es limitado, pero lo publicado permite ser optimista (Monte, 2011). A continuación, exponemos algunos de los estudios iniciales en esa nueva dirección. 


\subsection{Líneas de investigación en curso}

Uno de los retos a los que se enfrentan los publicitarios y los anunciantes es el de crear marcas memorables, consideradas como lo suficientemente importantes y relevantes para que sean almacenadas en la memoria del consumidor a largo plazo, ya que una marca memorable tendrá más posibilidades de ser elegida. Esto fue demostrado por Young (2009), quien realizó un experimento para probar que los anuncios más exitosos son los que consiguieron un mayor número de momentos recordados.

Como resultado de este experimento, y de otros, se están iniciando investigaciones con el objetivo de encontrar formas de crear marcas relevantes. Dada la complejidad de la memoria, la mayor parte de estudios implican una investigación parcial, relacionada con alguna de sus características o tipos. Por ejemplo, en relación con la memoria implícita, que acabamos de describir en el apartado anterior, se están enfocando varias líneas de investigación que a continuación detallamos:

- Una característica importante de la memoria implícita es que la información se puede adquirir inconscientemente, $o$ incluso puede ser adquirida mientras las personas presentan unos niveles de atención bajos (recordemos que las estancias cerebrales relacionadas con la memoria están siempre activas). Esto hace posible que las personas aprendan y que la formación de la memoria se produzca sin esfuerzo consciente (Heath, 2001). Este factor es importante para los estudios en el ámbito de la investigación publicitaria, ya que es posible que un anuncio tenga un efecto sobre la marca aunque los niveles de atención consciente sean bajos y que, incluso cuando éste no es ni recordado de forma explícita, sí puede tener una influencia en las elecciones de marca, tal y como Heath describió en su teoría denominada Low Involvement Processing (LIP). En esta teoría, Heath destaca que las asociaciones implícitas que el consumidor establece con la marca son las que ayudan a tomar las decisiones de consumo. Afirma, a su vez, que la mayoría de estas asociaciones pasan desapercibidas por la mente consciente, pero tales asociaciones no logran tampoco explicar satisfactoriamente la eficacia de los estímulos o cómo funciona la persuasión publicitaria. Lo que sí describe esta teoría es que las asociaciones implícitas construidas alrededor de las marcas sí poseen una influencia sobre nuestras decisiones y percepciones, incluso desde el punto de vista de la atención inconsciente.

- Algunos de los primeros estudios realizados sobre la memoria implícita y su relación con el reconocimiento de marca (Brand recognition) han sido desarrollados con éxito por Butler y Berry. Éstos demostraron en sus experimentos que el priming hacia marcas desconocidas en tareas de memoria implícita puede dar resultados similares a los observados cuando se comparan con los resultados de las tareas de memoria explícita. Señalaron, además, que el 
priming sólo tuvo un efecto significativo cuando los participantes juzgaban las marcas de baja involucración, lo cual enfatiza la importancia del procesamiento inconsciente de la memoria implícita (Butler, Berry, 2001).

- Otro ejemplo que podemos ver en el ámbito de la publicidad se encuentra en el estudio de los efectos de la comunicación online y en cómo medir su eficacia, ya que parece ser que la mayoría de las veces los anuncios son ignorados mientras se está navegando por Internet. Y, sin embargo, según lo que estamos argumentando acerca de la memoria y la atención, estos anuncios deberían tener algún efecto sobre la marca y la actitud hacia ella y su consideración. Existen estudios que tratan de responder a esta hipótesis, como por ejemplo, el de Chan Yun Yoo de la Kentucky University. Este estudio experimental examinó los efectos de los procesos inconscientes sobre anuncios online manipulando los niveles de atención (directa o indirecta). Los resultados sugieren que una exposición previa en Internet de un anuncio favorece el priming a partir de la memoria implícita, construyendo una actitud más favorable hacia la marca anunciada, independientemente del nivel de atención prestado en la visualización del anuncio. Los participantes no recordaban haber visto el anuncio explícitamente pero, sin embargo, tenían mayor probabilidad de incorporar la marca anunciada a su set de consideración, que los que no habían estado expuestos inconscientemente al anuncio (Chan Yun Yoo, 2008).

Además de estos interesantes estudios sobre memoria implícita, es importante destacar, aunque sea brevemente, otras líneas de investigación que se están desarrollando en la actualidad desde el neuromarketing sobre otros aspectos de la memoria igualmente influyentes:

- Rossiter y su equipo de investigadores (2001) realizaron un experimento centrado en la búsqueda de las áreas del cerebro responsables de la transferencia de los estímulos visuales en la memoria, con el fin de descubrir qué partes de los anuncios son los más memorables y determinar, así, si existía algún patrón cortical en las distintas escenas en particular cuando éstas eran más recordadas. Estos investigadores encontraron que el hemisferio izquierdo estaba más activo durante la codificación de la memoria, mientras que el hemisferio derecho se encargaba de la recuperación de tales recuerdos.

- Este hecho fue corroborado por Silberstein y Nield (2008). Ellos encontraron que el correlato neuronal entre la memoria a largo plazo y la preferencia de la marca ocurría en el lado izquierdo del lóbulo prefrontal lateral, resultado consistente con los estudios de Rossiter.

- Los estudios realizados por investigadores de la Università di Roma, en la que la investigadora Astolfi y su equipo (Astolfi et al, 2009), utilizaron EEG (electroencefalogramas) para describir las diferencias en la actividad cerebral 
entre personas que recordaban un anuncio específico y personas que no lo recordaban. En sus resultados, los procesos cerebrales involucrados durante la observación de anuncios de televisión demostraban que sí que existían diferencias en la actividad cerebral entre los participantes que recordaron y los que no recordaron los anuncios publicitarios.

- Un campo realmente interesante es el que se plantea si la comunicación puede cambiar nuestras memorias experienciales, también llamadas ilusiones asociadas por la memoria (Plassmann et al., 2007). Investigaciones utilizando nuevas herramientas de medición biométrica parecen validar que así es, ya que la respuesta de los individuos es muy sensible a los procesos provocados por la integración del contexto.

- Muchos de estos estudios se definen como exploratorios, sin embargo implican grandes avances. Por ejemplo, Ambler et al. (2004) and Braeutigam et al. (2004) utilizaron en este experimento MEG (magnetoencefalógrafo) para medir la correlación neuronal del proceso de decisión en el punto de venta. Los sujetos debían seleccionar una marca de entre las tres que se presentaron. Las decisiones se tomaron en una media de un segundo y se describían dos estadios de decisión. En el primero, se dieron procesos neuronales asociados con el reconocimiento de un problema y el recuerdo (memory recall). El segundo estaba relacionado con la elección concreta. Se describió una actividad cerebral diferente cuando la marca era familiar que cuando eran productos de marcas que los sujetos no conocían. La familiaridad de la marca fue un indicador útil para predecir el producto que elegirían, y también lo fue el tiempo empleado para tomar la decisión, pues cuando la marca era conocida, la decisión era más rápida. Sin duda, más experimentos como estos nos ayudarían a entender mejor la naturaleza del proceso de decisión, así como el rol de la memoria y de las emociones asociado a tal proceso cognitivo.

- En el área digital también se están realizando estudios que pueden revolucionar la manera actual de vender publicidad online, que suele ser a través de la cuantificación de impresiones. Esto es así gracias a investigaciones que han podido comprobar los efectos de la variación del tiempo de exposición de anuncios online en la memoria (Goldstein, McAfee, Suri, 2011).

- Incluso en el área de investigación publicitaria para videojuegos, tiene cabida la investigación que incorpora la medición biométrica de la respuesta de los participantes. Por ejemplo, en un estudio reciente, se pudo observar que el impacto de las medidas fisiológicas, utilizando electrocardiografía, fueron las más relevantes en relación a la memoria (Hernández y Minor, 2011).

Desde marcas a productoras de Hollywood, incluso editores, ya incorporan estudios de neuromarketing para profundizar en sus estudios sobre el consumidor. No todos tratan directamente sobre la memoria, pero indirectamente siempre tiene algún tipo de influencia en los consumidores. Microsoft investiga las interacciones 
de los usuarios con los ordenadores, Frito Lay ha estudiado el cerebro femenino para conocer mejor a la mujer, Google mide la efectividad de los diferentes tipos de video, Daimler busca conocer aquellos atributos del automóvil que sugieren rostros humanos por su mayor vinculación al centro de recompensa del cerebro, etc. (Alvarez, 2011).

\subsection{Líneas de investigación futura}

Es de esperar que la investigación sobre la memoria desde la neurociencia cognitiva siga aportando mayor conocimiento, nuevos ejemplos, la validación de hipótesis existentes y la creación de nuevas. Especialmente interesante será observar y seguir su desarrollo, incluso desde áreas tan innovadoras como la neuroquímica y la neurogenética, que atenderán mejor a las diferencias entre individuos, punto sin duda crucial para explicar gran parte de estos procesos.

Sin embargo, lo más importante para el neuromarketing y la industria publicitaria es ser capaces de poder explotar los avances ya existentes, y los nuevos que estén por venir, a su beneficio. Saber aprovechar todo este nuevo conocimiento para conseguir estrategias y creatividades más eficaces, así como herramientas de medición más fiables y útiles. Para ello es fundamental establecer puentes entre la universidad, la investigación académica y las empresas vinculadas a la actividad publicitaria (agencias, consultoras, institutos de investigación y anunciantes). Es importante que se establezcan líneas de investigación conjunta o, al menos, que exista un diálogo para que la publicidad no se quede atrás en los nuevos descubrimientos científicos, que podrían cambiar muchos de los presupuestos teóricos con los que ésta trabaja.

Como punto de partida, teniendo en cuenta que el ser humano está diseñado tanto para recordar como para olvidar, deberíamos aceptar que olvidar no es un problema desde el punto de vista biológico y, por tanto, convivir con ello de un modo más natural. Por esa razón, sugerimos que, desde el ámbito publicitario, quizás podría estudiarse tanto el recuerdo como el olvido. Una área de estudio concreta podría ser la exploración sobre la relación del olvido con la fuente de recuerdo, uno de los tipos de olvido más frecuentes (Schacter, 2001), y cuál es la relación entre ese olvido y aspectos como la credibilidad.

También sería muy interesante investigar las memorias falsas. Como punto de partida se podrían utilizar los estudios de Braun-Latour sobre la influencia del feeling of familiarity para generar recuerdos incorrectos. Sus teorías se basan en experimentos en los que se presentan estímulos visuales, en forma de anuncio gráfico, que incluyen personajes muy conocidos pero incorrectos en ese contexto, y que, posteriormente, cuando se habla de experiencias infantiles en dichos contextos se incorporan los personajes incorrectos en la historia como si hubieran estado presentes en realidad (Braun-Latour, 2004). 
Sobre la creación de falsas memorias, cabe destacar otro estudio que realizaron Rajagopal y Montgomery. Estos investigadores diseñaron el experimento para estudiar los efectos del visionado de anuncios de producto con alta o baja imaginaria mental para la generación de falsas memorias. Los autores demostraron que los participantes expuestos a un anuncio de alta imaginaria mental experimentaron la creencia falsa de haber probado el producto que el anuncio publicitaba. También demostraron que la función de tales creencias relacionadas con la experiencia de producto fortalecía la actitud hacia aquel producto concreto, llamaron al fenómeno el "efecto de experiencias falsas". Imágenes vívidas dieron a los voluntarios recuerdos de degustación de un producto que no existía. (Rajagopal y Montgomery, 2011). Otra área importante de desarrollo futuro viene de la mano de la incorporación y el uso de técnicas biométricas en el estudio de la memoria en relación con la investigación publicitaria. Actualmente, se están empezando a utilizar estas técnicas para identificar las áreas del cerebro que están involucradas en la memoria y en cómo crear recuerdos. Técnicas tales como el encefalógrafo (EEG) son muy útiles para dar, a tiempo real, información precisa (milisegundos) sobre la actividad en las diferentes regiones del cerebro mientras la información está siendo procesada. El EEG se puede utilizar para monitorizar la naturaleza de la activación cerebral de un consumidor a medida que se presentan marcas conocidas a partir de los estímulos publicitarios y, además, se pueden utilizar y combinar tales hallazgos con los métodos de investigación más tradicionales (como pueden ser los cuestionarios) para averiguar por qué las marcas son tan memorables y tratar de emular, posteriormente, su efecto sobre otra marca no tan conocida. Por ejemplo, las primeras averiguaciones sobre los mecanismos de la memoria en relación con la actividad del cerebro mostraron que un incremento en los procesos de la memoria se daba cuando existía correlación con la actividad en la onda alfa en el cerebro (Rothschild y Hyun, 1990), mostrando una correspondencia consistente entre la formación de la memoria y la actividad cerebral.

Una manera de proseguir con estas investigaciones en un futuro, será poder, en condiciones de pre-test, predecir si un determinado estímulo publicitario será o no será recordado. Y, más importante, determinar qué escenas serán más recordadas que otras. Creemos que estos experimentos pueden llegar a ser relevantes por numerosas razones, quizás la más importante sea que, gracias a ellas, se empieza a poder explicar cómo generar recuerdos para saber cuáles son los momentos -y qué tipología de escenas o qué elementos de la ejecución- más susceptibles de ser recordados. En esta línea de investigación, la herramienta BioQual que la empresa multinacional de investigación TNS ha lanzado recientemente -combinando información biométrica (EEG, GSR respuesta galvánica de la piel y el Eye Tracking) con técnicas de investigación cualitativa como el Focus group - atienden a esta área de investigación. De Balanzó, Ohme y Eising sugieren en su artículo que cuando una determinada escena obtiene una relevancia positiva (o negativa) en los resultados del EEG y una alta activación (GSR) será la escena que tenga más posibilida- 
des de ser recordada, ya que la área de la corteza pre-frontal esta también relacionada con procesos de la memoria. Aunque un mayor número de experimentos son necesarios para poder dar cuenta del recuerdo publicitario a través de estas técnicas y algoritmos, este es un camino prometedor: el poder encontrar la manera de llegar a predecir las escenas que serán más recordadas y, sobre todo, llegar a poder explicar por qué unas lo son más que otras. (Balanzó, Ohme, Eising, 2010).

Finalmente, es muy posible que se generen más estudios desde el neuromarketing que avalen la relación entre la memoria y el resto de procesos cognitivos, especialmente con aquellos fundamentales para la publicidad, como son la atención y la emoción. Por ejemplo, en relación con la emoción, ya se han producido avances de la mano del neurocientífico Antonio Damasio. Damasio ofrece una nueva perspectiva del rol de la memoria, ya que, estudiando la relación de la memoria con la emoción, abre las puertas a una nueva área de investigación. Su punto de partida fundamental es la hipótesis del marcador somático, validando que todos los objetos son emocionalmente competentes, ya sean estímulos reales como recordados por la memoria. Un hallazgo fundamental de cara a su aplicación en el ámbito de la actividad publicitaria y el marketing es el concepto que utilizan los autores, el "emotional packet" (de Balanzó, Serrano y Scamell-Katz, 2010). El emotional packet contiene toda la información relacionada con las marcas y los atributos emocionales vinculados al producto. Como estos autores describen, este paquete se convierte en un atajo cuando elegimos marcas en el punto de venta, facilitando el proceso de la toma de decisiones. La creación del emotional packet empieza con aquello que el consumidor quiere o necesita de una categoría determinada y después cómo éste define las marcas en su mente. Este paquete está formado por todas las actividades de marketing, tanto aquellas que impulsan los recuerdos analíticos de alta atención, y que serían descritas si al consumidor se le pidiera que hablara de la marca, como aquellas producidas por un proceso de baja implicación utilizando muy poca memoria de trabajo, lo cual significa que es muy pobre interpretando mensajes. (Scamell-Katz y Balanzó, 2012).

\section{Conclusiones}

En el artículo se han revisado los principales hallazgos sobre las características de la memoria, obligándonos a reflexionar sobre su definición actual. En línea con los nuevos avances de las neurociencias, definimos la memoria bajo las siguientes características: no literal, activa, flexible, adaptativa, dinámica, subjetiva, que reinterpreta, olvida y se equivoca.

La memoria también debemos entenderla como un complejo y sofisticado sistema de sistemas. Esta memoria no unitaria puede clasificarse bajo dos tipologías básicas: memoria a corto y a largo plazo, y memoria explícita e implícita. Vimos que la memoria a corto plazo es la capacidad de mantener una determinada canti- 
dad de información reciente por un período corto de tiempo. Es muy útil para el rápido procesamiento cognitivo de los estímulos percibidos, pero posee una actividad espacio-temporal más limitada.

Por el contrario, la memoria a largo plazo dijimos que ocupa un área más grande del cerebro en comparación con la memoria a corto plazo y que participa activamente en la vida diaria (es decir, vocabulario, navegación o la misma memoria de trabajo). No obstante, como aspecto muy relevante para el ámbito publicitario, ésta no es totalmente fiable y los recuerdos que hay en ella pueden ser manipulados y reordenados por los recuerdos y/o las percepciones de otros. De ahí la maleabilidad y subjetividad que describimos cuando definimos la memoria.

Sobre la dimensión explícita e implícita, recogimos que la memoria explícita describe la información que una persona puede declarar, como son los hechos y el conocimiento de los acontecimientos vitales específicos que le acaecen a lo largo de su vida. Al contrario, la memoria implícita incluye todas aquellas memorias que no pueden rescatarse conscientemente, las que surgen de modo involuntario, así como las que agrupan «conocimientos generales», como, por ejemplo, tocar un instrumento, el lenguaje, la sintaxis o ir en bicicleta. De hecho, un aspecto muy importante de esta dimensión es que no somos conscientes de que tenemos tales conocimientos y los utilizamos de forma automática. Esta dimensión está muy relacionada con algunos de los mecanismos que subyacen en la toma de decisiones.

A raíz de la diferenciación de tales tipologías de la memoria, surge la necesidad de contar con herramientas de investigación que, en la actividad e investigación publicitaria, puedan contemplar y explicar tales dimensiones. Y de esta distinción surge la necesidad de complementar las técnicas de investigación tradicional con las técnicas de neuroimagen y neurofisiológicas que permitan poder cuantificar y cualificar todos esos procesos biológicos e inconscientes que explican gran parte del comportamiento humano.

El mercado del neuromarketing se encuentra aún en su infancia, pero en los próximos años estará en posición de ofrecer nuevos e interesantes avances científicos y profesionales (Monge y Fernández, 2011). A partir de nuestra interpretación de los avances existentes, y con la expectativa de los que están por venir, pensamos que deberíamos reconsiderar el papel de la memoria en la eficacia publicitaria. Esta nueva perspectiva debe seguir nutriéndose de los nuevos avances y tecnologías desarrollados por la neurociencia cognitiva y el neuromarketing. En este artículo, hemos definido algunas líneas de investigación a corto y a largo plazo, pero, en línea con el pensamiento de Damasio, cada vez sabemos más sobre el cerebro, pero todavía queda tanto camino por recorrer, que siempre sabremos más que el año anterior y mucho, mucho más, que la década anterior (Damasio, 2010). De ahí que debamos ser cautos y precisos en la manera cómo incorporemos los nuevos conocimientos a las prácticas publicitarias desde cualquiera de sus ámbitos. Hacen falta más investigaciones que avalen estos y otros estudios ya que, sin duda, tienen la posibilidad de cambiar la manera cómo entendemos la actividad publicitaria y es- 
tamos seguros que aportarán una mayor rigurosidad científica a la investigación y la creación publicitaria, pero es fundamental que neurocientíficos y publicitarios trabajen juntos (Plassmann et al., 2007) para que se pueda llegar a avanzar tanto como prometen las nuevas tecnologías y los últimos avances científicos.

\section{Referencias bibliográficas}

AlvareZ, R. (2011): Neuromarketing, Madrid, Pearson Educación.

AmBler, T., [et al.] (2004): «Salience and Choice: Neural Correlates of Shopping Decisions». Psychology \& Marketing, vol. 21(4), 247-261.

Astolfi, A., [et al.] (2009): «Brain activity during the memorization of visual scenes from TV commercials: An application of high resolution EEG and steady state somatosensory evoked potentials technologies». Journal of Physiology, vol. 103, num.6, 333-341.

BALANZÓ, C. de.; SABATÉ, J. (2007): «Neurociencias y publicidad: la nueva frontera de la persuasión». Trípodos, $\mathrm{n}^{\circ}$ extra 2007, 909-923.

BALANZÓ, C. de.; SerRano, N.; SCAMELl-KatZ, S. (2010): «Damasio: a starting point for integrating neuroscience findings into retail research». En: ESOMAR WORLD RESEARCH CONFERENCE. Odyssey 2010: the changing face of market research: congress papers. Amsterdam: ESOMAR, 2010, 465-483.

BALANZÓ, C. de.; OHME, R. ;EISING, H. (2011): « Predicting brand decisions through emotional engagement». En: ESOMAR WORLD RESEARCH CONFERENCE. Amsterdam: ESOMAR, 2011, 31-44.

BICKART, K. [et al.] (2011): «Amygdala volume and social network size in humans». Nature Neuroscience, vol. 14, issue 2, 163-164.

BRAUN-LATOUR, K.; LATOUR, M. (2004): «Assessing the long-term impact of a consistent advertising campaign on consumer memory». Journal of Advertising, vol. 33 num. 2. 49-61.

Braun-Latour, K.; ZaltMan, G. (2006): «Memory Change: An intimate measure of Persuasion». Journal of Advertising Research, 46 (1), 57-72.

BUTLER, L.T.; BERRY, D.C. (2001): «Transfer effects in implicit memory and consumer choice». Applied Cognitive Psychology, 15, 587-601.

CHAN YUN YOO (2008): «Unconscious processing of web advertising: effects on implicit memory, attitude toward the brand and consideration set». Journal of interactive marketing, vol. 2, num. 2.

CowAN, N. (2000). «The magical number 4 in short-term memory: A reconsideration of mental storage capacity». Behavioral and Brain Sciences, 24, 87-185.

DAMASIO, A. (2003): Looking for Spinoza: joy, sorrow and the feeling brain, Orlando, Harcourt Books.

- (2005): Descartes' error: emotion, reason, and the human brain, New York, Penguin Books.

- (2010): Self Comes to Mind, Constructing the Conscious Brain, London, Random House.

DUDAI, Y. (2004): Memory from A to Z; keywords, concepts and beyond, New York, Oxford University Press.

Eichenbaum, H. (2003): Neurociencia cognitiva de la memoria, Barcelona, Ariel. 
Franzen, G.; BouWMan, M. (2001): The mental world of brands: mind, memory and brand success. Henley-on-Thames, World Advertising Research Centre.

GoldsteIn, D.; McAfeE, P.; SuRI, S. (2011): «The Effects of Exposure Time on Memory of Display Advertisements». Proceedings of the $12^{\text {th }}$ ACM Conference on Electronic Commerce.

HEATH, R. (2001): «Low involvement processing: a new model of brand communication». Journal of marketing communication, vol. 7 (2001), $\mathrm{n}^{\mathrm{o}}$ 1, 27-33.

- (2000): «Low involvement processing: a new model of brands and advertising». International journal of advertising, vol. 19 (2000), n 3, 287-298.

HERNANDEZ, M.; MINOR, M. (2011): «Investigating the effect of arousal on brand memory in advergames», Qualitative Market Research: An International Journal, vol. 14, $\mathrm{n}^{\mathrm{0}} 2$, 207-217.

Howes, M. (2007): Human Memory. Thousand Oaks, Sage Publications.

Miller, G. (1956): «The Magical Number Seven, Plus or Minus Two», Psychological Review, vol. 63, 81-97.

Monge, S.; FernandeZ, V. (2011): «Neuromarketing: Tecnologías, Mercado y Retos». Pensar la Publicidad, vol. 5, No.2, 19-42.

PLASSMANN, H., [et al.] (2007): «What can advertisers learn from neuroscience?». International Journal of Advertising, 26 (2), 151-175.

RAJAGOPAL, P. ;MONTGOMERY, N.V.(2011): «I Imagine I Experience, I Like: The False Experience Effect». Journal of Consumer Research, 38.

RATEY, J. A. (2004): User's Guide to the Brain, London, Abacus.

ROTHSCHILD, M.L.; HYUN, Y.J. (1990): «Predicting memory for components of TV commercials from EEG». Journal of Consumer Research, 16(4), 472-478

RossitER, J.R., [et al.] (2001): «Brain-imaging detection of visual scene encoding in longterm memory for TV commercials». Journal of Advertising Research, 41(2), 13-21.

SAMPEDRO, M.P. (1966): «Plasticidad Neural». En: El cerebro intimo, Barcelona, Ariel.

SCAMELL-KATZ, S; BALANZÓ, C. de. (2012): «Leyendo la mente del comprador ». Tu cerebro lo es todo, Barcelona, Plataforma Editorial.

SCHACTER, D. (1996): Searching for Memory: the brain, the mind, and the past, New York, Basic Books, Perseus.

- (2001): The Seven Sins of Memory. How the Mind Forgets and Remembers, Boston, New York, Houghton Mifflin Company.

SILBERSTEIN R.; NIELD,G. «Brain activity correlates of consumer brand choice shift associated with television advertising». International Journal of Advertising, Vol. 27, No. 3, 2008, 359-380.

SQUIRE, L.R. (1992): «Memory and the hippocampus: a synthesis from findings with rats, monkeys and humans». Psychological Review, 99(2), 195-231.

YounG, C. (2009): Ad response tests show how attention connects to memory, ADMAP magazine.

Recibido: 4 de noviembre de 2011

Aceptado: 6 de noviembre de 2012 